\title{
Perceptual Grouping through Competition in Coupled Oscillator Networks
}

\author{
Martin Meier, Robert Haschke and Helge J. Ritter * \\ Bielefeld University, Neuroinformatics Group \\ 33615 Bielefeld - Germany
}

\begin{abstract}
In this paper we present a novel approach to model perceptual grouping based on phase and frequency synchronization in a network of coupled Kuramoto oscillators. Transferring the grouping concept from the Competitive Layer Model (CLM) to a network of Kuramoto oscillators, we preserve the excellent grouping capabilities of the CLM, while dramatically improving the convergence rate, robustness to noise, and computational performance, which is verified in a series of artificial grouping experiments.
\end{abstract}

\section{Introduction}

The ability to robustly group related perceptual items to form higher-order concepts is crucial for many cognitive tasks. Exploiting the recurrent dynamics of neurons, the Competitive Layer Model (CLM) [3] has proven to solve a broad spectrum of complex grouping tasks in a very robust fashion - even in the presence of strong noise. Amongst others, these tasks include segmentation of cell images [4], grouping of object contours in edge images [5], as well as motion segmentation [6]. However, a major drawback of the CLM for real-world applications, is its high demand for computational resources: The network converges slowly and each update step is costly. Furthermore, the network can only hardly escape from a reached optimum, when the grouping dynamics is changed.

Hence, inspired by the fast synchronization ability of coupled oscillator networks $[7,1,2]$, we transfer the grouping principles of the CLM to a network of Kuramoto oscillators [7] in order to improve the computational performance. In the new model, each oscillator represents a distinct input feature from an arbitrary feature domain. The coupling strengths between the oscillators are based on the compatibility of the corresponding features. Similar features have a positive compatibility, therefore the corresponding oscillators phase-lock and form a perceptual group, which repels dissimilar features by means of negative couplings. The Kuramoto model has been investigated in many variations [8, 9] and we refer the interested reader to this work.

In the following sections, we shortly outline the principles of the CLM and introduce our approach to transfer them to a network of coupled Kuramoto oscillators. In section 4 we evaluate both approaches with regard to the grouping quality and convergence speed in the presence of increasing levels of noisy connections. Finally, the results are discussed.

\footnotetext{
${ }^{*}$ This work has been conducted within and funded by the German collaborative research center "SFB 673: Alignment in Communication" granted by DFG.
} 


\section{The CLM for Perceptual Grouping}

The CLM consists of $N \times L$ neurons which are arranged in $L$ layers. Neurons are indexed column wise with $m=1, \ldots, N$ describing the position in each layer and $\alpha=1, \ldots, L$ denoting the layer index. A single neuron's activity is therefore denoted as $x_{m, \alpha}$. The neurons in each layer are coupled with a symmetric interaction function $f\left(v_{m}, v_{n}\right)=f\left(v_{n}, v_{m}\right)=f_{m, n}$ which describes the compatibility between two features $v_{m}$ and $v_{n}$. They are additionally coupled with a winner takes all (WTA) circuit in each column to assure that only one neuron in each column becomes active. Combining the lateral interaction and columnar WTA circuit, the recurrent CLM dynamics can be written as:

$$
\dot{x}_{m, \alpha}=-x_{m, \alpha}+\sigma\left(J\left(1-\sum_{\beta=1}^{L} x_{m, \beta}\right)+\sum_{n=1}^{N} f_{m, n} x_{n, \alpha}\right) .
$$

Here $J\left(1-\sum_{\beta} x_{m, \beta}\right)$ represents the WTA competition weighted by the constant $J$, and $\sigma(\cdot)$ is a linear threshold function. The lateral interaction is expressed as $\sum_{n} f_{m, n} x_{n, \alpha}$, which calculates the support for the feature at position $m$ from all other features $n$ in a given layer $\alpha$. For a more comprehensive overview, we refer to [5].

\section{Transfer to Network of Coupled Kuramoto Oscillators}

The oscillator model replaces each CLM column - composed from $L$ neurons representing the grouping result for a given feature $v_{m}$ - with a single oscillator of the Kuramoto type [7]. Actually, we employ the extended approach [8] where the coupling between oscillators is determined by a symmetric matrix $M_{m, n} \equiv$ $f\left(v_{m}, v_{n}\right)$ instead of a constant gain factor $K$. Hence, the phases $\theta_{m}$ of the oscillators evolve according to the following update rule:

$$
\dot{\theta}_{m}=\omega_{m}+\frac{K}{N} \sum_{n=1}^{N} f\left(v_{m}, v_{n}\right) \cdot \sin \left(\theta_{n}-\theta_{m}\right) .
$$

The frequency of an oscillator is described with $\omega_{m}$. The interaction function $f$ is limited to the interval $[-1,1]$, where -1 and +1 represent strongest dissimilarity resp. similarity of features.

To resemble the CLM layer architecture, the oscillator frequencies are limited to discrete values $\omega_{\alpha}=\alpha \cdot \omega_{0}$, where $\alpha \in\{1, \ldots, L\}$ denotes the group index following the CLM notation where $\alpha$ denotes the group/layer index. To achieve a clustering of similar features to the same discrete frequency $\omega_{\alpha}$, the frequency $\omega_{m}$ of each oscillator is updated employing the cosine similarity between the phases of the oscillators which is mapped to the interval $[0,1]$ to preserve the sign of the coupling strengths $f\left(v_{m}, v_{n}\right)$. Hence, the frequencies are updated according to:

$$
\omega_{m}=\omega_{0} \cdot \underset{\alpha}{\operatorname{argmax}}\left(\sum_{n \in \mathcal{N}(\alpha)} f\left(v_{m}, v_{n}\right) \cdot \frac{1}{2}\left(\cos \left(\theta_{n}-\theta_{m}\right)+1\right)\right),
$$




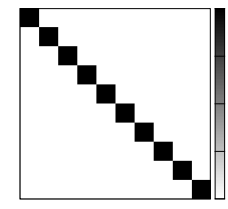

(a) $0 \%$ inverted.

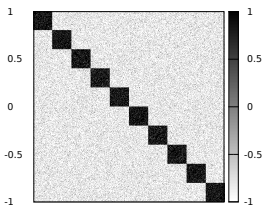

(b) $10 \%$ inverted.

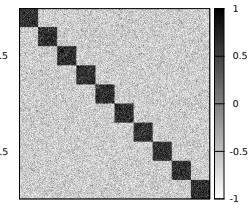

(c) $20 \%$ inverted.

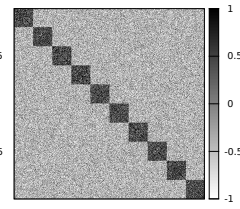

(d) $30 \%$ inverted.

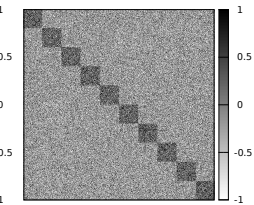

(e) $40 \%$ inverted.

Fig. 1: Visualization of interaction matrices with different amounts of inverted interaction values while preserving the symmetry. Each matrix represents ten groups with positive coupling strength within each group (black) and negative coupling strength with the other groups (white).

where $\mathcal{N}(\alpha)$ denotes the set of oscillators with frequency index $\alpha$, i.e. forming the current perceptual group indexed by $\alpha$. This update ensures that oscillators representing similar features will both phase-lock and converge to identical frequencies. Eq. (3) also boosts the phase-locking process, because synchronized phases do not tend to desynchronize anymore. Contrarily, oscillators representing dissimilar features will spread both in phase and frequency.

With an appropriate interaction function, a network which follows the update equations (2), (3) produces a stable grouping result already after a few update steps. The final grouping result is determined by oscillator subsets $\mathcal{N}(\alpha)$ having common frequency indices $\alpha$.

The computational complexity of this model is reduced compared to the CLM. Because each of the $N$ features is represented by $L$ individual neurons in the CLM model, we yield a complexity of $\mathcal{O}(N \cdot L \cdot(N+L))$. By representing each feature with a single oscillator only, the oscillator network reduces the complexity to $\mathcal{O}(N \cdot(N+L))$, which is especially superior for many groups $L$.

\section{Evaluation}

In the following evaluation, the proposed oscillator model is compared to the CLM. First an overview of the simulation settings is given. Subsequently, the grouping quality and convergence speed of both models are compared w.r.t. different amounts of noise in the connection strengths.

\subsection{Simulation Settings}

For the evaluation process, we used a CLM and an oscillator network with similar topologies. The CLM was created for $10^{3}$ features and 100 layers, leading to a total of $10^{5}$ neurons. Accordingly, the oscillator network was created with $10^{3}$ oscillators, each representing a single feature and all possible groupings by 100 discrete frequencies. To determine the grouping result with respect to noise, we employed randomly perturbed, binary interaction matrices $F \equiv f_{m, n}$. These matrices represent ten groups of features, where each group comprises 100 features. Noise was introduced by inverting the sign of the coupling strength between two 


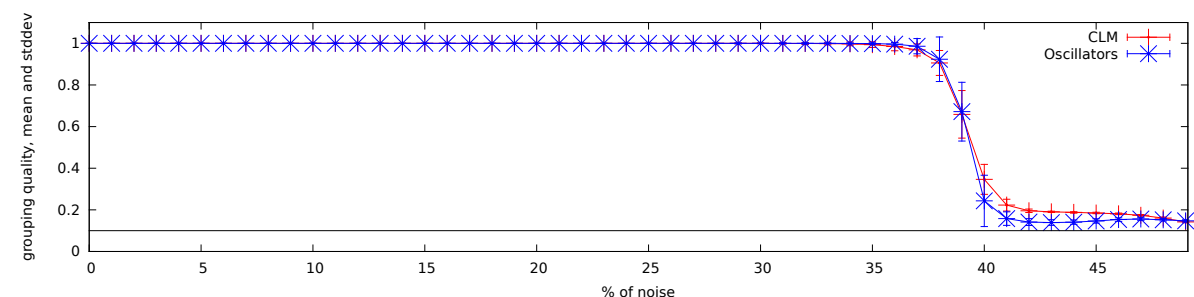

Fig. 2: This figure shows the grouping quality of the CLM and the oscillator network with respect to different amounts of inverted interaction values. Both methods show good results for up to $35 \%$ of inverted interaction values.

features while preserving the symmetry of the matrix. Example matrices for different levels of perturbations are shown in Fig. 1, where couplings of +1 and -1 are visualized as black resp. white dots.

For each level of perturbation, ranging from $0 \%$ to $49 \%$, we simulated 500 trials with 1000 update steps each. For both networks, the CLM and Kuramoto model, a single update step comprises an update of all neurons (1) resp. oscillators (2), (3). Please note, that the CLM has $L=100$ neurons for each feature, which is represented by a single oscillator in the Kuramoto model. Hence, the oscillator network is computationally much less demanding. However, we refrained from measuring the absolute computation time to yield comparable results w.r.t. convergence speed (measured in update steps here).

Both models are randomly initialized at the beginning of each trial. In case of the CLM, every neuron got a small positive activity. The oscillators in the network were uniformly distributed over all possible frequencies and initialized with random phases in the range $[0,2 \pi]$.

\subsection{Grouping Quality}

In the first part of the evaluation, the grouping quality of the CLM and the oscillator network is compared w.r.t. ground truth. To this end, we employ the quality measure $Q$ used in [5] to evaluate the grouping quality of the CLM. Given target labels $t_{m} \in\{1, \ldots, T\}$ for each feature $v_{m}$, these labels are compared to the grouping result $a_{m} \in\{1, \ldots, T\}$ of the CLM and the oscillator network, respectively. The grouping quality $Q$ is then calculated as

$$
Q=\frac{1}{N^{2}} \sum_{m}^{N} \sum_{n}^{N} q_{m, n}, \quad q_{m, n}=\left\{\begin{array}{lll}
1 & \text { if } & t_{m}=t_{n} \text { and } a_{m}=a_{n} \\
1 & \text { if } & t_{m} \neq t_{n} \text { and } a_{m} \neq a_{n} \\
0 & \text { else }
\end{array}\right.
$$

which yields a value in the interval $[0,1]$, with 1 representing a perfect grouping result. In Fig. 2 the average grouping quality over 500 trials is shown with respect to the level of noise. The simulation is stopped after 1000 update steps. For up to $35 \%$ of perturbed connections, both methods show more or less similar performance, but the CLM degrades more slowly for larger levels of noise, that 


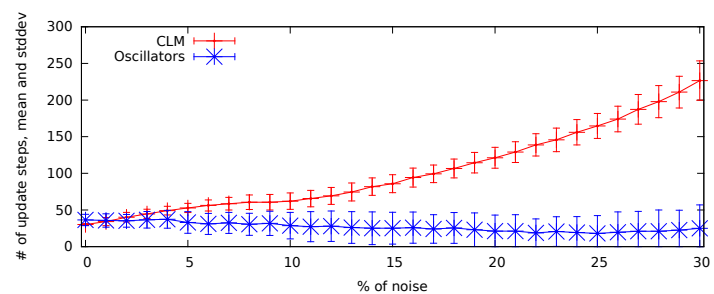

(a) $0 \%$ to $30 \%$ of interaction values inverted.

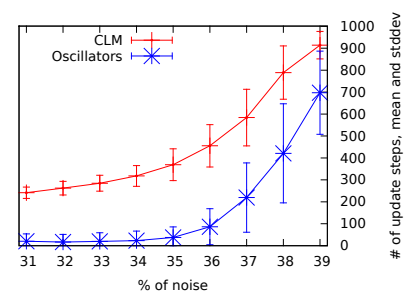

(b) $31 \%$ to $39 \%$.

Fig. 3: Here the mean number of update steps and standard deviation to achieve a grouping quality of $95 \%$ are shown. Please note that the y-axis differs between Fig. 3(a) and Fig. 3(b).

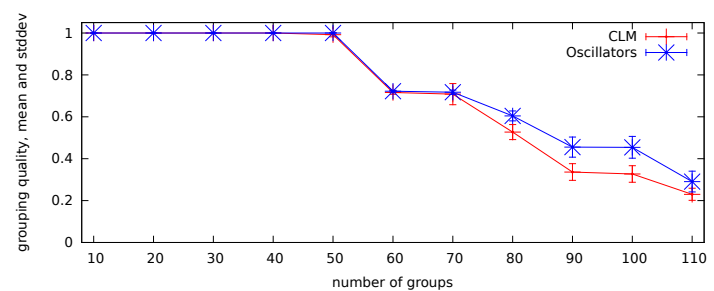

(a) Grouping quality vs. number of target groups.

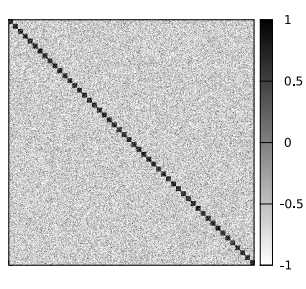

(b) 50 groups.

Fig. 4: This figure shows the grouping quality with respect to the number of different classes in the interaction matrix. The amount of noise is set to $20 \%$ in all cases. In Fig. 4(a) the quality is shown for 10 to 110 target groups. Fig. 4(b) shows an interaction matrix with 50 groups.

is for cases with $40 \%$ and more noise. The black line in Fig. 2 indicates the pure chance level.

\subsection{Grouping Speed}

Another important factor is the number of updates each model takes to find a correct grouping result. In Fig. 3 the average number of update steps (with standard deviation) are shown, which are needed to achieve a grouping result of at least $95 \%$ correct assignments. Up to a noise level of 33\%, the oscillator network needs an almost constant amount of updates to achieve a sufficient grouping result, while the CLM shows a quadratic performance degradation. After this mark, both methods exhibit an exponential increase of update steps and also the standard deviation increases in both cases. This may be due to the decreased sampling size, because trials not achieving the 95\% quality threshold within 1000 update steps where discarded from the evaluation. Although the oscillator network has a bigger variance in the number of update steps, it outperforms the CLM already at a noise rate of $5 \%$. 


\subsection{Influence of Number of Target Groups}

To gain insight into the impact of the number of target groups, we varied the number of groups from 10 to 110 while keeping a constant amount of $20 \%$ noise. The remaining settings are similar to the previous evaluations. Both methods show very good results for up to 50 target groups, as shown in Fig. 4. After that mark, the grouping quality decreases. Except for the case with 110 groups, one factor could be the limitation to 1000 steps, because the smaller number of features per group also reduces the attracting characteristic of compatible features. The oscillator model shows better results with a larger number of groups, which can be explained due to the overall faster grouping speed which was found in the previous evaluation.

\section{Conclusion and Outlook}

We presented a novel approach for perceptual grouping based on synchrony in an oscillator network and compared it to a well known technique, the Competitive Layer Model. The evaluation revealed that the proposed approach has the same perceptual grouping capabilities with respect to noisy input data, but achieves an adequate grouping result with less update steps while having a smaller computational complexity. The Kuramoto model also offers the possibility of a dimensionality reduction, where each oscillator is not updated with respect to each other oscillator but to their mean phase. This property transfered to the here introduced discrete frequencies offers another possibility to further decrease the computational complexity and will be subject to an upcoming evaluation. Additionally, we strive to investigate the behavior of the presented oscillator model with respect to disturbances in the input data. As a dynamical system, it should be able to recover from external disturbances. This will also be an interesting point for further investigations.

\section{References}

[1] D.A. Paley, N.E. Leonard, R. Sepulchre, D. Grunbaum, and J.K. Parrish. Oscillator models and collective motion. Control Systems, IEEE, 27(4):89-105, 2007.

[2] P. Pezuk, J. Mohawk, T. Yoshikawa, M. Sellix, and M. Menaker. Circadian organization is governed by extra-scn pacemakers. Journal of biological rhythms, 25(6):432-441, 2010.

[3] H. Ritter. A spatial approach to feature linking. In Int. Neural Network Conference, 1990.

[4] Nattkemper, Wersing, Schubert, and Ritter. A neural network architecture for automatic segmentation of fluorescence micrographs. Neurocomputing, 48(1):357-367, 2002.

[5] H. Wersing. Spatial feature binding and learning in competitive neural layer architectures. Cuvillier, 2000.

[6] J. Steffen, M. Pardowitz, J. Steil, and H. Ritter. Neural competition for motion segmentation. Proc. ESANN, 2010.

[7] Y. Kuramoto. Chemical oscillations, waves, and turbulence. Dover Publications, 2003.

[8] H. Hong and S.H. Strogatz. Kuramoto model of coupled oscillators with positive and negative coupling parameters: An example of conformist and contrarian oscillators. Physical Review Letters, 106(5):54102, 2011.

[9] P.S. Skardal and J.G. Restrepo. Hierarchical synchrony of phase oscillators in modular networks. Physical Review E, 85(1):016208, 2012. 